\title{
The mycotoxin deoxynivalenol facilitates allergic sensitization to whey in mice
}

\author{
M Bol-Schoenmakers ${ }^{1,4}$, S Braber ${ }^{1,4}$, P Akbari ${ }^{1,2}$, P de Graaff ${ }^{1}$, M van Roest ${ }^{1}$, L Kruijssen ${ }^{1}$, JJ Smit ${ }^{1}$, \\ BCAM van Esch $^{2,3}$, PV Jeurink ${ }^{2,3}$, J Garssen ${ }^{2,3}$, J Fink-Gremmels ${ }^{1}$ and RHH Pieters ${ }^{1}$
}

Intestinal epithelial stress or damage may contribute to allergic sensitization against certain food antigens. Hence, the present study investigated whether impairment of intestinal barrier integrity by the mycotoxin deoxynivalenol (DON) contributes to the development of whey-induced food allergy in a murine model. C3H/HeOuJ mice, orally exposed to DON plus whey once a week for 5 consecutive weeks, showed whey-specific IgG1 and IgE in serum and an acute allergic skin response upon intradermal whey challenge, although early initiating mechanisms of sensitization in the intestine appeared to be different compared with the widely used mucosal adjuvant cholera toxin (CT). Notably, DON exposure modulated tight-junction mRNA and protein levels, and caused an early increase in IL-33, whereas CTexposure affected intestinal $\gamma \delta$ T cells. On the other hand, both DON- and CT-sensitized mice induced a time-dependent increase in the soluble IL-33 receptor ST2 (IL-1R1) in serum, and enhanced local innate lymphoid cells type 2 cell numbers. Together, these results demonstrate that DON facilitates allergic sensitization to food proteins and that development of sensitization can be induced by different molecular mechanisms and local immune responses. Our data illustrate the possible contribution of food contaminants in allergic sensitization in humans.

\section{INTRODUCTION}

Over the last decades, the prevalence of food allergies has increased to $6 \%$ in children and $3 \%$ in adults. ${ }^{1}$ As no therapies are available yet, treatment of food allergies relies primarily on allergen avoidance to prevent food-induced adverse reactions in sensitized individuals. Food allergies are the result of abnormal immunologic responses to food antigens, resulting in antigen-specific IgE-mediated reactions, with symptoms varying from mild skin itching to severe and potentially life-threatening anaphylaxis. ${ }^{2}$ Despite extensive research, the mechanism of initiation of allergic sensitization to food antigens remains poorly understood.

In most models of food allergy, the mucosal adjuvant cholera toxin (CT) seems indispensable for provoking allergic sensitization to food proteins in mice. One of the important mechanisms related to the adjuvant effect of CT, is the disruption of the intestinal epithelial cell layer by breakdown of adherens molecules via a cAMP-driven mechanism. ${ }^{3}$ This fits closely with the immunological concept that sensitization requires a danger signal, such as tissue damage.
It is already known that in skin and lung, disruption of the epithelial barrier could induce allergic sensitization. For instance, the proteolytic activity of house dust mite allergens disrupts the tight-junction network in lung epithelium. This leads to release of endogenous danger signals and cytokines by epithelial cells which initiate activation of dendritic cells (DC), followed by the series of events resulting in IgE synthesis by B cells. ${ }^{4}$ Also in the skin, mild epithelial damage is accompanied by the induction of systemic IgE. In this case, the damaged skin epithelial cells upregulate stress molecules and alarmins such as IL-25, IL-33, and TSLP, which results in migration and activation of epidermal $\gamma \delta$ T cells., ${ }^{5,6}$

In line with the observations in skin, in vivo studies in mice by our group and others have shown migration and activation of intraepithelial lymphocytes (IEL) including $\gamma \delta \mathrm{T}$ cells in response to CT exposure. ${ }^{7,8}$ These and additional results, demonstrating that blocking of the $\gamma \delta$ TCR enhances allergic manifestations, ${ }^{7}$ are reminiscent of involvement of epithelial damage in sensitization to food proteins. In support of this, we

\footnotetext{
${ }^{1}$ Institute for Risk Assessment Sciences, Utrecht University, Utrecht, The Netherlands. ${ }^{2}$ Utrecht Institute for Pharmaceutical Sciences, Utrecht University, Utrecht, The Netherlands and ${ }^{3}$ Nutricia Research, Utrecht, The Netherlands. Correspondence: M Bol-Schoenmakers (m.bol-schoenmakers@uu.nl)

${ }^{4}$ These authors contributed equally to this work. 
found that epithelial damage induced by diclofenac enhances CT-induced allergic sensitization to peanut in mice. ${ }^{9}$ Moreover, it was recently shown that the alarmin uric acid is a critical signal for the induction of peanut allergy in mice, ${ }^{10}$ suggesting that cellular damage is indeed involved in the onset of allergic sensitization.

Based on these findings, we hypothesize that other substances that cause epithelial cell stress or damage may act as an adjuvant to potential food allergens. Hence, we investigated whether the trichothecene mycotoxin deoxynivalenol (DON), known as biologically active metabolite that impairs intestinal barrier function by direct effects on intestinal epithelial cells, ${ }^{11}$ may act as a danger signal. DON produced by Fusarium species is among the most frequently detected contaminants in cereal-based foods, resulting in regular human exposure. $^{12}$

This paper describes the disruption of the intestinal junctional network by DON to facilitate sensitization in a murine allergy model in comparison with CT-induced allergic sensitization. In addition, the effect of DON and CT on intestinal epithelial integrity and consecutive activation of local immune cells is assessed to get more insight into molecular mechanisms of allergic sensitization.

\section{RESULTS}

\section{The mycotoxin DON facilitated allergic sensitization}

Mice exposed to DON plus whey displayed a significant increase in whey-specific IgG1, IgG2a, and IgE after 5 weeks, compared with mice exposed to DON or whey alone (Figure 1a-c). Furthermore, whey-specific restimulation of splenocytes from DON plus whey-sensitized mice revealed a specific induction of the Th2 cytokines IL- 5 and IL-13 but not of the Th1 cytokine IFN- $\gamma$ (Figure 1d-f). As expected, CT induced allergic sensitization based on all measured parameters. Overall, the allergen-specific responses were significantly higher in CT plus whey-sensitized mice in comparison with DON plus whey-sensitized mice.

In addition, DON plus whey-treated mice showed an acute allergic skin response upon intradermal injection with whey, as shown by a significant increase in ear thickness compared with mice exposed to DON or whey alone (Figure 1g). In three out of eight mice, the increase in ear thickness was accompanied by mild anaphylactic shock symptoms (Figure 1h), while one mouse showed a decrease in body temperature after intradermal challenge (data not shown). The acute allergic skin response in CT plus whey-sensitized mice was significantly enhanced compared with DON sensitization, and was accompanied by anaphylactic shock symptoms in six out of eight mice (Figure $\mathbf{1 h}$ ) and a decrease in body temperature in all mice (data not shown).

Serum mast cell protease-1 (MCPT-1) levels, a measure for mucosal mast cell degranulation, were not elevated after oral allergen challenge in DON plus whey-sensitized mice in contrast to CT plus whey-sensitized mice, in which an increase was observed (Figure 1i). In addition, MCPT-1 ${ }^{+}$cells were increased in the proximal small intestine in CT plus whey-sensitized mice $24 \mathrm{~h}$ after oral whey challenge, but not in control mice or DON plus whey-sensitized mice. MCPT- ${ }^{+}$cells in CT-exposed mice were mainly present in the crypts (Supplementary Figure S1 online).

Although a higher dose of DON $(200 \mu \mathrm{g}$ per mouse instead of $100 \mu \mathrm{g}$ per mouse) caused side effects, this dose did induce an acute allergic skin reaction accompanied by shock symptoms, a decrease in body temperature, and enhanced MCPT-1 serum levels after oral sensitization (Supplementary Figure S2).

\section{DON altered epithelial permeability resulting in allergen translocation in vitro}

As a consequence of alterations in the function and expression of tight-junction proteins, DON is able to impair transepithelial electrical resistance (TEER) of epithelial Caco- 2 cell monolayers in vitro and to increase paracellular tracer transport. ${ }^{11}$ In the present study, $1.2 \mu \mathrm{g} \mathrm{ml}^{-1}(4.2 \mu \mathrm{M})$ DON exposure resulted in a significant decrease in TEER (Figure $2 \mathbf{b}$ ), which facilitated the translocation of beta-lactoglobulin, one of the major allergenic whey proteins, from the apical to the basolateral side of the Caco-2 monolayer (Figure 2a). In contrast, beta-lactoglobulin was not translocated across the Caco-2 monolayer after exposure to $100 \mu \mathrm{g} \mathrm{ml}^{-1} \mathrm{CT}$ (Figure 2a), despite the small but significant decrease in TEER (Figure $2 \mathbf{b}$ ).

The expression pattern of tight and adherens junction proteins claudin $(\mathrm{CLDN}) 3$ and E-cadherin in the Caco-2 monolayer showed a disturbed cellular distribution in DONexposed cells, with loss of the typical honey-rate structure and increased cytosolic expression. DON affected CLDN4 to a lesser extent, and the disturbed cellular distribution was not observed in CT-exposed cells (Figure 2c). Hoechst staining of monolayers confirmed an equal distribution of Caco- 2 cells independent of exposure conditions (Supplementary Figure S3).

\section{In vivo exposure to DON resulted in altered expression of} barrier function-associated molecules in duodenum

To gain more insight into intestinal changes that might contribute to whey sensitization, gene expression of molecules related to epithelial damage was assessed $6 \mathrm{~h}$ after a single oral exposure to CT or DON. mRNA expression of the tight-junction molecules CLDN2 and -3 (Figure 3a,b) was upregulated in the duodenum after DON but not after CT exposure. In addition, the mRNA expression of CLDN1 and $-4, \mathrm{ZO} 1$ and OCLN remained unaffected (data not shown). Furthermore, DON significantly upregulated the gene expression of E-cadherin in the duodenum, whereas CT did not significantly alter E-cadherin expression (Figure 3c). mRNA expression of the stress-associated molecules RAE-1 (Figure 3d) and HMGB-1 (data not shown) was not altered by DON or CT in the duodenum, whereas in the ileum RAE-1 mRNA expression was increased by CT, but not by DON. In addition to gene expression, CLDN3 and E-cadherin protein levels were determined by western blot analysis in duodenum 6 and $24 \mathrm{~h}$ after a single oral exposure to DON or CT. DON reduced CLDN3 and E-cadherin protein levels in the proximal 

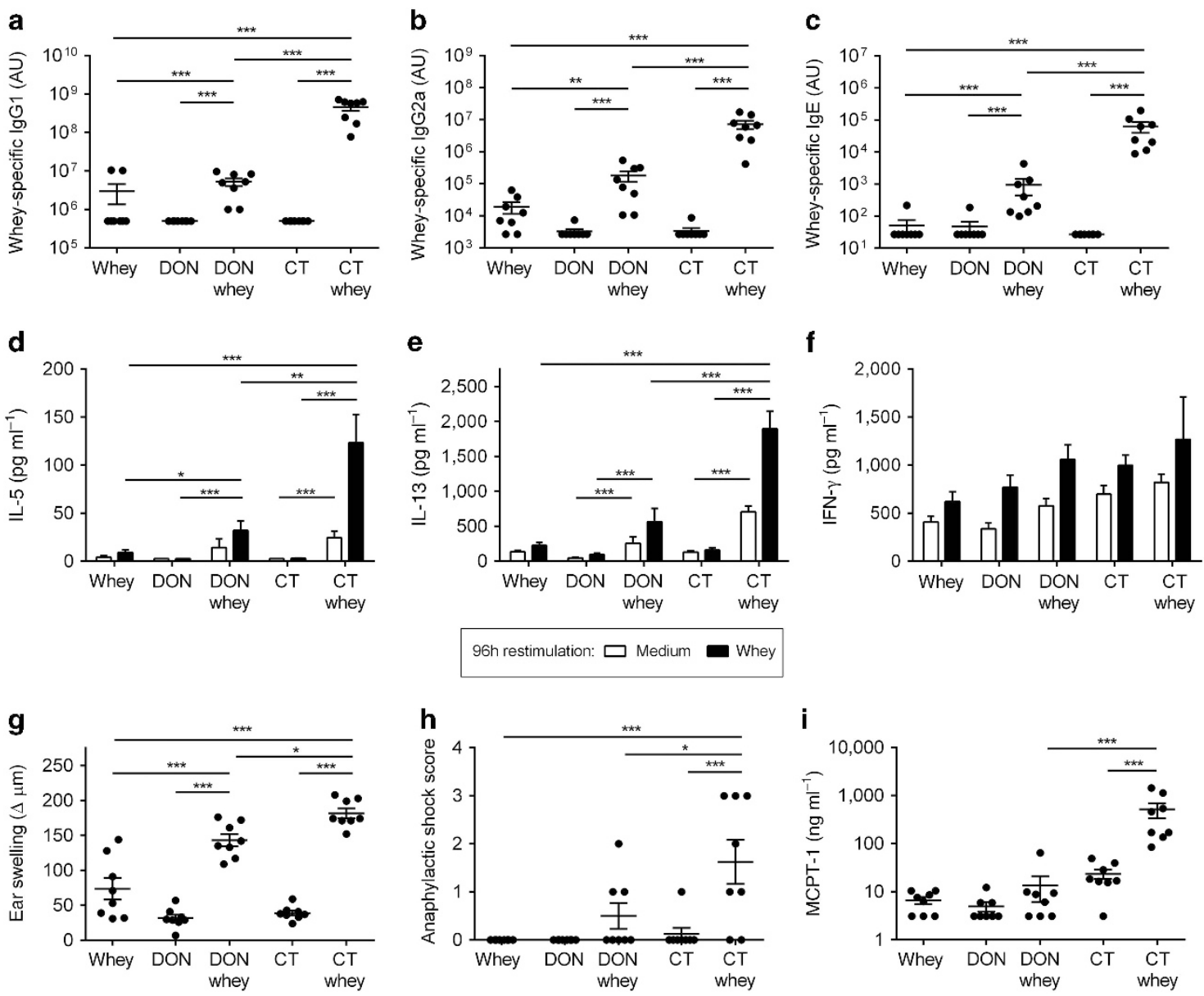

Figure 1 Capacity of deoxynivalenol (DON) to facilitate allergic sensitization. Female $\mathrm{C} 3 \mathrm{H} / \mathrm{HeOuJ}$ mice $(n=8)$ were orally exposed to whey, DON or cholera toxin (CT) alone, to whey plus DON or to whey plus CT once a week for 5 consecutive weeks. (a-c) Whey-specific IgG1, IgG2a, and IgE in serum were determined by ELISA. (d-g) Levels of IL-5, IL-13, and IFN $\gamma$ were determined in culture supernatant of ex vivo restimulated splenocytes from sensitized mice. $(\mathbf{g}, \mathbf{h})$ The acute allergic skin response in sensitized mice was determined by measuring ear swelling $1 \mathrm{~h}$ after intradermal whey challenge, and accompanying anaphylactic score was determined after $45 \mathrm{~min}$. (i) Mast cell protease-1 (MCPT-1) levels in serum, collected $30 \mathrm{~min}$ after an oral whey challenge, were determined by ELISA. Data are presented as mean \pm s.e.m. ${ }^{*} P<0.05,{ }^{* \star} P<0.01$, ${ }^{* * *} P<0.001$; one-way ANOVA followed by Bonferroni's multiple comparison test (after log transformation of data except for ear swelling and anaphylactic shock score).

small intestine at both time points in comparison with control treatment, whereas CT only reduced these expression levels after $24 \mathrm{~h}$ (Figure $\mathbf{3 e}-\mathbf{g}$ ). The decreased protein levels, however, were more pronounced after a DON gavage in comparison with CT.

Despite changes in CLDN3 and E-cadherin, no increase in the in vivo uptake of the major whey protein $\beta$-lactoglobulin was observed after a single oral exposure to DON or CT (Supplementary Figure S4).

\section{Alterations in barrier-associated molecules is accompanied by IL-33 and innate lymphoid cells type 2 induction}

To investigate whether changes in CLDN3 and E-cadherin affect local immune factors, we assessed IL-33 in the intestine. DON- but not CT-exposed mice showed a statistically significant increase in IL-33 expression in the duodenum, both in gene expression and in protein level (Figure 4a,b). This increased intestinal IL-33 in DON-exposed mice was accompanied by an increase in soluble IL-33 receptor ST2 in the intestine (Figure 4c) and serum (Figure 4d) $6 \mathrm{~h}$ after DON exposure. However, mice exposed to DON or CT plus whey once a week for 5 consecutive weeks showed a gradual increase in soluble ST2 in serum (Figure 4e). Remarkably, by displaying data per time point (Supplementary Figure S5), it is clear that DON- but not CT-exposed mice already demonstrated significantly increased levels of soluble ST2 levels after the first and second sensitization.

To investigate whether the local presence of IL-33 in the intestine was associated with activation of innate lymphoid cells type 2 (ILC2), the percentage and activation status of ILC2 in lamina propria were assessed using flow cytometry. ILC2 cells were identified as $\mathrm{CD} 45^{+} \mathrm{CD} 127$ (IL-7R) ${ }^{+}$but lineage-negative cells expressing ST2 (Figure 4h). Both DON and CT enhanced the percentage of ILC2 cells $24 \mathrm{~h}$ after a single oral dose in combination with whey (Figure 4f). ST2 median fluorescence intensity was not significantly altered by DON or CT (Figure 4g). 

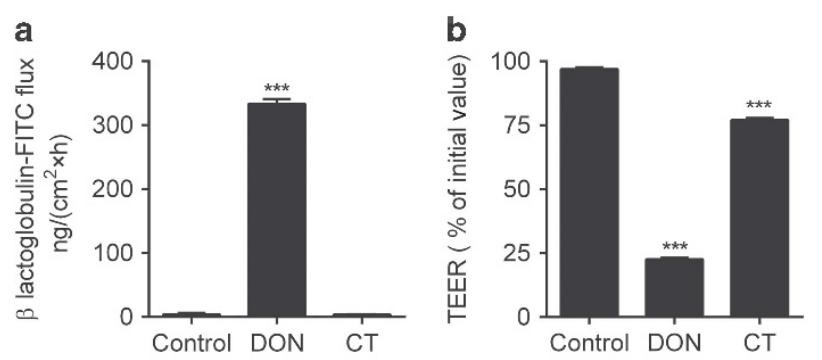

c

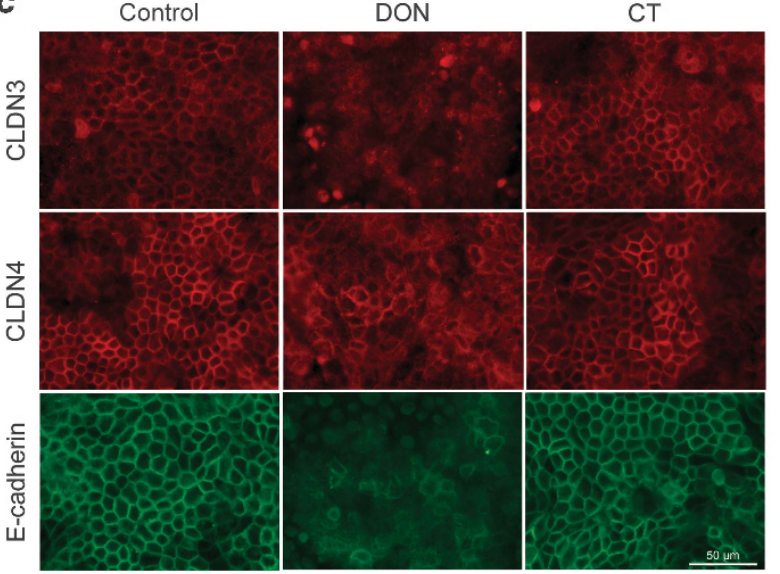

Figure 2 Effect of deoxynivalenol (DON) and cholera toxin (CT) on Caco-2 monolayer integrity. Caco-2 cells were grown on inserts and exposed to DON or CT at the apical and basolateral side. (a) After $24 \mathrm{~h}$ exposure, the paracellular transport of $\beta$-lactoglobulin-FITC (fluorescein isothiocyanate) from apical to basolateral compartment was determined and (b) transepithelial electrical resistance (TEER) was measured. (c) Cellular expression of CLDN3, CLDN4, and E-cadherin in Caco-2 monolayer was assessed by immunofluorescent staining. Data are presented as mean \pm s.e.m. from three separate experiments, each performed in triplicate. ${ }^{* *} P<0.001$; one-way ANOVA followed by Bonferroni's multiple comparison test.

\section{DON and CT differently altered intestinal cell subsets}

Flow-cytometric analysis of cells isolated from the whole small intestine was performed $24 \mathrm{~h}$ after a single oral dose of DON plus whey or CT plus whey, as changes in intestinal cell subsets likely occur at a later time point compared with transcriptional changes. Results displayed no significant changes in the number of isolated lymphocytes due to exposure (Figure 5a). In line with our previous findings, ${ }^{7}$ CT significantly reduced the number of $\gamma \delta$ T cells in intestinal cell compartments, as well as the relative presence of $\gamma \delta$ T cells within the IEL (Figure 5b). In contrast, DON did not alter the number of $\gamma \delta$ T cells in the different intestinal cell compartments (Figure 5b).

Assessment of DC subsets showed a small increase in $\mathrm{MHCII}^{+} \mathrm{CD}_{11 \mathrm{c}^{+}} \mathrm{DC}$ in different intestinal cell compartments after DON exposure, both in mucosal $\mathrm{CD}_{103}{ }^{+}$and in monocyte-derived $\mathrm{CD}_{11 \mathrm{~b}^{+}}$subsets of DC (Figure 5c). In contrast, CT significantly enhanced the percentage of DC in mesenteric lymph nodes (MLN) with a comparable contribution of $\mathrm{CD}_{103}{ }^{+}$and $\mathrm{CD}_{11} \mathrm{~b}^{+}$expressing $\mathrm{DC}$ (Figure 5c). Elevated MHCII membrane expression on individual DC in the MLN after CT exposure pointed toward the presence of more mature DC in the MLN, and immune activation (Figure 5d). However, CT exposure did not significantly enhance the expression of the activation marker CD86, associated with T-cell activation. Furthermore, OX40L expression, associated with activation of Th2 cells, was also not elevated on DC in the MLN $24 \mathrm{~h}$ after a single oral dose.

\section{DISCUSSION}

This study investigated whether epithelial cell stress or damage and the subsequent innate immune response contribute to allergic sensitization. Hence, we compared CT, often used as a tool to unravel the mechanisms of allergic sensitization, with DON. The mycotoxin DON is among the most frequently detected contaminant of cereal-based foods, including breakfast cereals, ${ }^{12}$ whereas CT is not present in the human food chain. Different studies demonstrated that DON is able to impair intestinal barrier function, ${ }^{11,13}$ and is therefore a suitable candidate to investigate the contribution of epithelial damage on allergic sensitization.

We report that DON as well as CT facilitates allergic sensitization to whey proteins, indicated by increased serum levels of whey-specific IgE, an acute allergic skin response upon allergen challenge and by the production of IL-5 and IL-13 in whey-restimulated splenocytes.

However, the mechanism by which DON supports allergic sensitization appears different from the mechanism of the more generally used mucosal adjuvant CT. Although CT reduced the TEER in Caco-2 cell monolayer, it did not disturb epithelial integrity like DON, as the distribution of tight and adherens junction molecules stayed unaltered, and no in vitro $\beta$-lactoglobulin translocation occurred. The decrease in TEER induced by CT is probably the result of changes in ion channels due to the adenylate cyclase-mediated rise in cAMP concentration in epithelial cells upon CT ligation of the gangliosideGM1 receptor. ${ }^{14}$ Others showed that repeated CT exposure during $40 \mathrm{~h}$ affected adherens junction molecules in Caco-2 cells, ${ }^{3}$ but effects seem very mild when compared with DON-induced barrier disruption. The proximal intestines of DON-exposed mice showed remarkable reduction in CLDN3 and E-cadherin protein levels accompanied by a compensatory increase in mRNA levels, which is indicative for alterations in the integrity of the intestinal epithelial cell layer, as demonstrated before. ${ }^{11}$ Although less pronounced, CT also reduced protein levels of CLDN3 and E-cadherin which is in line with findings by Guichard et $a l^{3}$ who demonstrated disorganized junctions after CT exposure in murine intestinal loops. However, not all junctional molecules seemed affected, as no alterations were observed in gene expression of CLDN1 and $-3, \mathrm{ZO} 1$, and OCLN after exposure to DON or CT. We previously showed that a single oral exposure to $25 \mathrm{mg} \mathrm{kg}^{-1}$ BW $(500 \mu \mathrm{g}$ per mouse) $\mathrm{DON}$ in $\mathrm{B} 6 \mathrm{C} 3 \mathrm{~F} 1$ mice resulted in increased translocation of $4 \mathrm{kDa}$ FITC (fluorescein isothiocyanate)-dextran ${ }^{11}$ because of altered barrier integrity. On the other hand, a single DON exposure to 1 or $10 \mathrm{mg} \mathrm{kg}^{-1} \mathrm{BW}$ did not alter intestinal permeability (unpublished results). In addition, the $\beta$-lactoglobulin uptake $6 \mathrm{~h}$ after exposure to $5 \mathrm{mg} \mathrm{kg}^{-1}$ BW DON (100 $\mu$ g per mouse) remained unaltered 

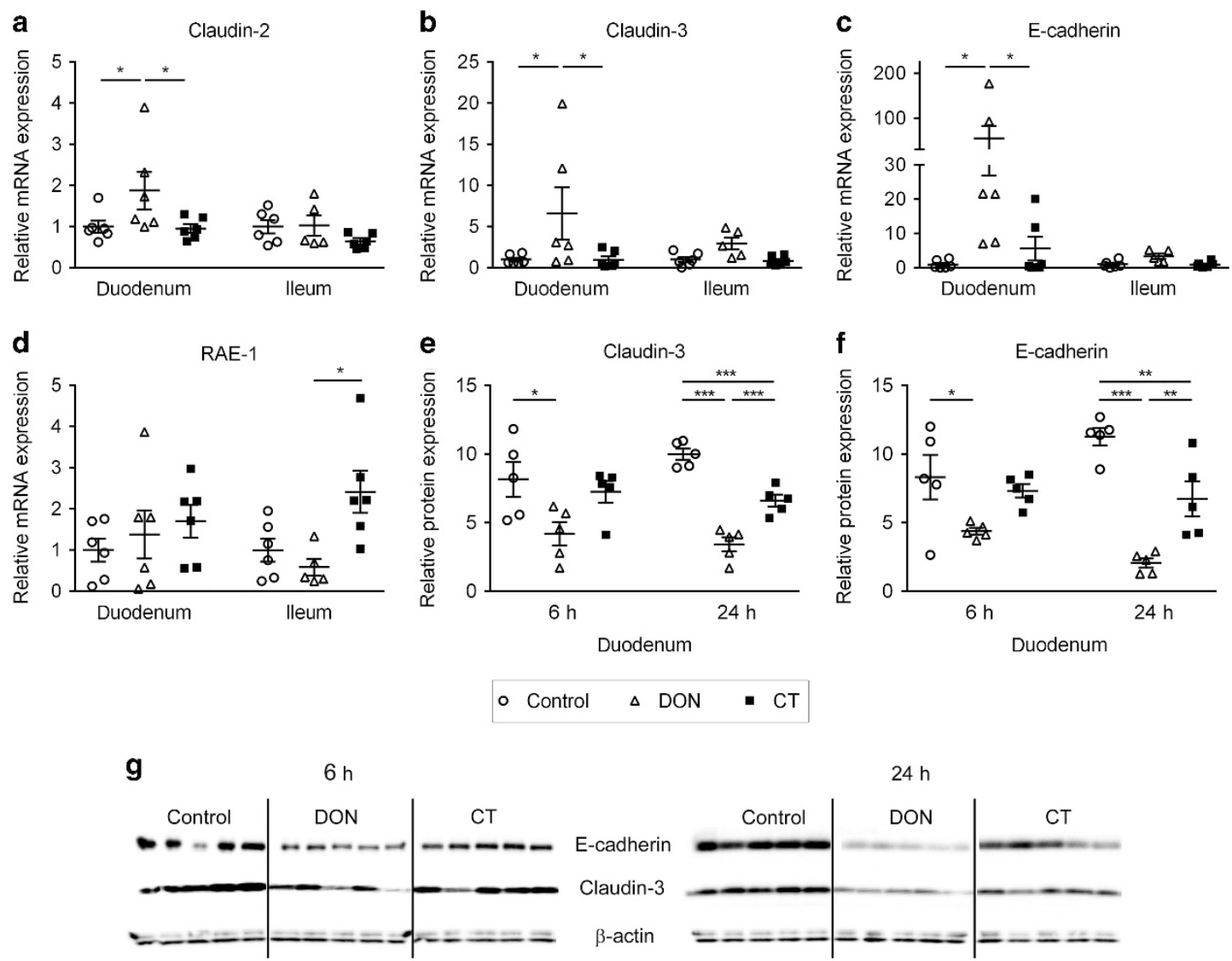

Figure 3 Effect of deoxynivalenol (DON) and cholera toxin (CT) on intestinal barrier integrity molecules. Mice were orally exposed to phosphatebuffered saline (PBS) (control), DON, or CT. After 6 or $24 \mathrm{~h}$, intestinal tissue was dissected and homogenized for qPCR analysis or western blotting. Relative mRNA expression (fold of control, normalized to $\beta$-actin) was determined for (a) CLDN2 (b) CLDN3, (c) E-cadherin, and (d) RAE-1 after $6 \mathrm{~h}$. Relative protein levels (fold of control, normalized to $\beta$-actin) were determined for (e) CLDN3 and (f) E-cadherin after 6 and $24 \mathrm{~h}$. (g) The immunoblot obtained with antibodies for E-cadherin (MW $98 \mathrm{kDa}$ ), CLDN3 (MW $22 \mathrm{kDa}$ ), and $\beta$-actin (MW $42 \mathrm{kDa}$, protein loading control). Note: membranes were cut to keep order of treatment groups similar to other panels. Data from 5-6 mice per treatment group and time point are depicted as mean \pm s.e.m. ${ }^{*} P<0.05$, ${ }^{\star *} P<0.01,{ }^{\star \star *} P<0.001$; one-way ANOVA followed by Bonferroni's multiple comparison test.

in comparison with vehicle-treated mice in the present study. Therefore, alterations in barrier-associated junctional molecules as induced by DON, and in to a lesser extent by CT, only in part results in impaired barrier integrity and translocation of allergens after a single exposure. In the course of the current food allergy study, we repeatedly exposed mice to whey plus $100 \mu \mathrm{g}$ DON per mouse to induce allergic sensitization. Although not assessed, it is possible that the intestinal permeability increases after cumulative DON or CT dosing. Barrier disruption facilitates translocation of allergens, which may contribute to allergic sensitization. Nevertheless, alterations in barrier-associated junctional molecules after a single dosage seem sufficient to change intestinal immune parameters that contribute to allergic sensitization.

Notably, DON but not CT resulted in rapid induction of IL-33 in the duodenum, whereas both increased ILC2 cell numbers in the lamina propria. IL-33 may originate from damaged or stressed intestinal cells. ${ }^{15,16}$ It has become clear that IL-33 is crucial for the induction of ILC2 and DC activation leading to subsequent type 2 immune responses. ${ }^{17,18}$ The intestinal immune cell subsets in our study also showed an increase in activated DC, especially in MLN after CT exposure, and in lamina propria lymphocytes (LPLs) and Peyer's patches (PPs) after DON exposure. Furthermore, it has already been shown that exposure to CT resulted in increased intestinal IL-33 levels accompanied by increased ILC2 and DC numbers in the peanut allergy mouse model. ${ }^{19}$ Unlike differences in IL-33 production by DON and CT, we found that both compounds potentiated ILC2 and DC in the intestine or MLN after one exposure, suggesting a role for these cells in the onset of allergic sensitization, which is in agreement with Chu et al. ${ }^{19}$ However, differences in extent may be related to differences in CT exposure strategy.

Remarkably, compared with CT plus whey, DON plus whey induced the soluble IL-33 receptor ST2 very early in the onset of sensitization, whereas from day 14 these levels were more comparable. We suggest that these early differences are due to the specific damaging effect of DON on the epithelial barrier. It is thought that soluble ST2 acts as a decoy receptor, resulting in sequestration and neutralization of IL-33 activity, thereby preventing excessive immune activation. ${ }^{20}$ Our observations therefore indicate that soluble ST2 might be a suitable 

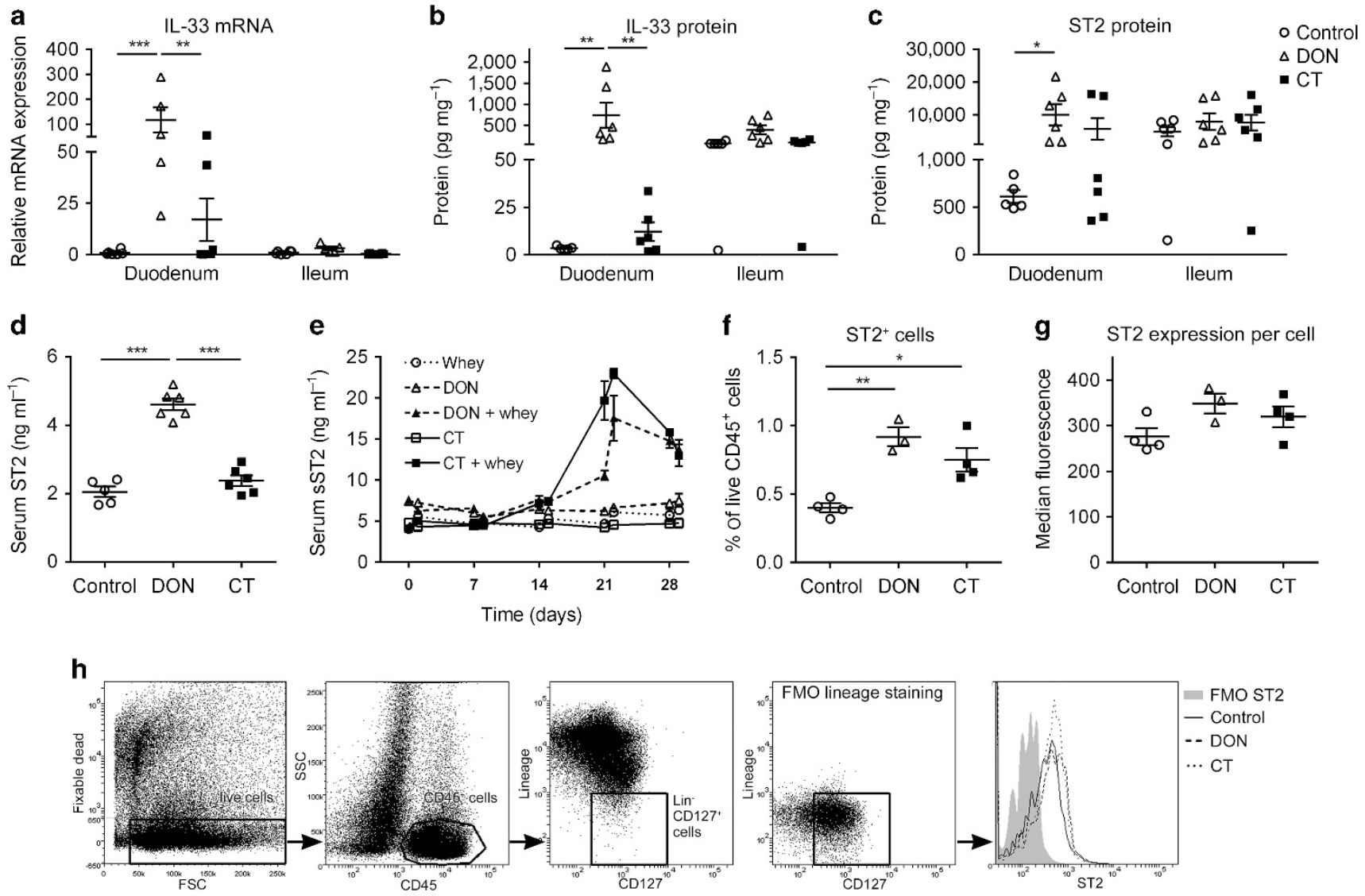

Figure 4 Activation of immune components in the intestine by deoxynivalenol (DON) and cholera toxin (CT). (a-c) Mice $(n=6)$ were orally exposed to phosphate-buffered saline (PBS) (control), DON, or CT, and $6 \mathrm{~h}$ later, intestinal tissue was dissected. One part was homogenized for determination of IL33 mRNA by qPCR and an adjacent part was homogenized for determination of IL-33 and ST2 protein by ELISA. (d). Soluble ST2 levels in serum samples $6 \mathrm{~h}$ after oral exposure to PBS (control), DON, or CT $(n=6)$. (e). Soluble ST2 levels in serum samples, collected $6 \mathrm{~h}(n=4)$ and $24 \mathrm{~h}(n=4)$ after each sensitization treatment as described in Figure 1. (f-h) Mice $(n=3-4)$ were orally exposed to whey alone (control), or to whey + DON or whey + CT and small intestines were dissected $24 \mathrm{~h}$ later for analysis of isolated innate lymphoid cells type 2 (ILC2) by flow cytometry. (f) Relative frequency of ST2 ${ }^{+}$ILC2 in the lamina propria (g) median fluorescent intensity (MFI) of ST2 on ILC2 in lamina propria. (h) Gating strategy. Data are depicted as mean \pm s.e.m. ${ }^{\star} P<0.05,{ }^{* \star} P<0.01,{ }^{* \star *} P<0.001$; one-way ANOVA followed by Bonferroni's multiple comparison test.

biomarker to detect allergic sensitization, and at least confirms the capacity of DON and CT to facilitate sensitization to whey proteins in mice.

Interestingly, in addition to the effect on ILC2, CT but not DON reduced $\gamma \delta \mathrm{T}$ cells in the small intestine. We propose that, comparable to skin, $\gamma \delta$ T cells respond to stressed epithelial cells by recognizing certain receptors on these cells, including RAE1 , and migrate to lymph nodes. ${ }^{5}$ However, the role of $\gamma \delta \mathrm{T}$ cells in CT-driven food allergic sensitization is still not understood since a recent paper showed that $\gamma \delta \mathrm{T}$ cells were not essential in allergic sensitization in CT-driven peanut allergy by using TCR $\delta \mathrm{KO}$ mice, ${ }^{21}$ whereas our group previously demonstrated a regulatory role for $\gamma \delta \mathrm{T}$ cells in allergic sensitization. ${ }^{7}$

Based on our data, we conclude that DON-induced epithelial cell stress results in a rapid increase in intestinal IL-33, which contributes to elevated intestinal numbers of ILC2 and DC. In comparison with DON, disturbing effects of CT on intestinal epithelium are different, since elevated ILC2 numbers are present without detectable IL-33, and in addition, activation and migration of $\gamma \delta \mathrm{T}$ cells and DC occurs. In both cases, these different cellular effects further initiate a Th2 response leading to allergic sensitization.

The exposure concentration of $100 \mu \mathrm{g}$ per mouse $\left(\sim 5 \mathrm{mg} \mathrm{kg}^{-1} \mathrm{BW}\right)$ as used in the present study exceeded the provisional maximum tolerable daily intake of $1 \mu \mathrm{g} \mathrm{kg}-1$ BW as set by the European Commission. ${ }^{22}$ However, several studies indicated that human exposure recurrently exceeded this safety level. ${ }^{23-25}$ Next to that, humans are likely not exposed to DON alone but to mixtures of group B trichothecenes and other mycotoxins. Recent data revealed that mycotoxins have synergistic and additive effects on epithelial cell viability in vitro. ${ }^{26,27}$ Other compounds capable of inducing epithelial cell damage or stress might also be able to elicit or aggravate allergic sensitization to certain food allergens in susceptible individuals. Therefore, this study emphasizes the need to further investigate the role of epithelial damage induced by environmental substances, including food contaminants, in relation to food allergy in humans. 


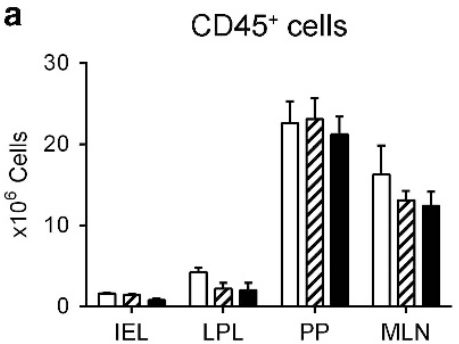

C

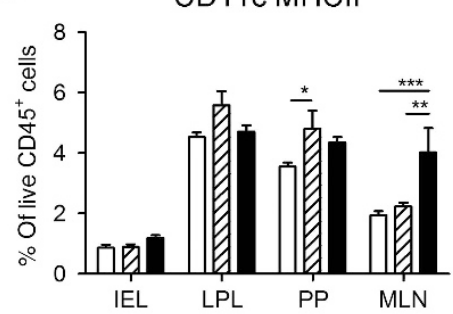

d

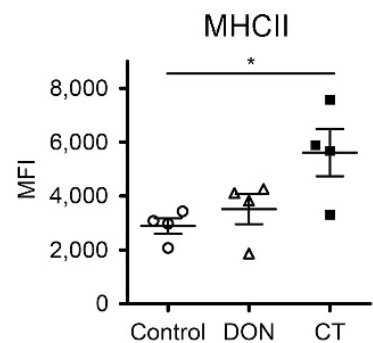

b

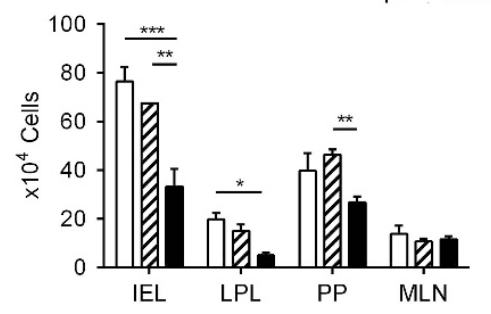

CD11C MHCII CD103
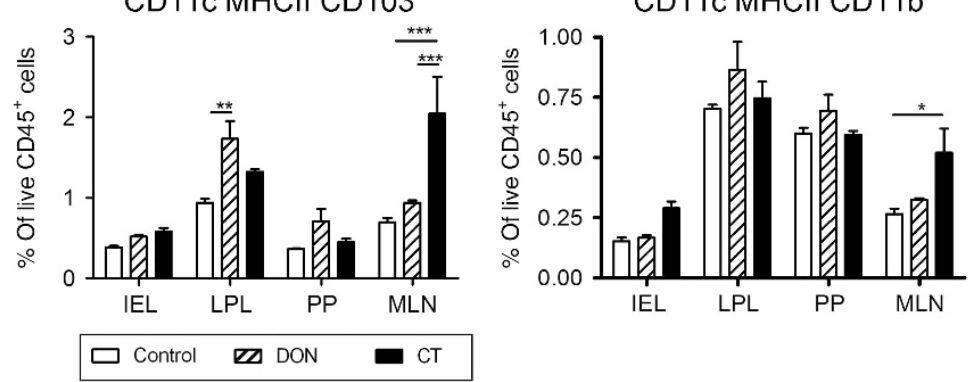

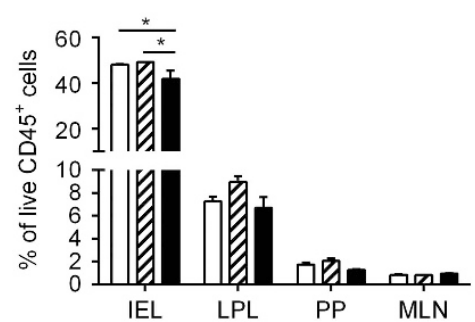

Marker expression on CD11 $\mathrm{c}^{+}$MLN cells

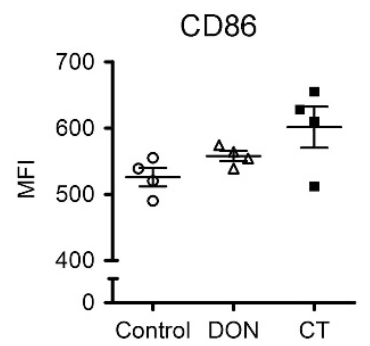

Figure 5 Analysis of intestinal immune cells after oral exposure to deoxynivalenol (DON) and cholera toxin (CT). Mice $(n=4)$ were orally exposed to whey alone (control), or to whey + DON or whey $+\mathrm{CT}$, and $24 \mathrm{~h}$ later, the small intestine and mesenteric lymph node (MLN) were dissected after which cells were isolated and characterized by flow cytometry. (a) Number of isolated lymphoid cells. (b) Number and relative frequency of $\gamma \delta \mathrm{T}$ cells. (c) Relative frequency of DC subsets. (d) Median fluorescent intensity (MFI) of maturation markers on CD11c ${ }^{+} \mathrm{DC}$ in the MLN. Data are depicted as mean $\pm \mathrm{s}$.e.m. ${ }^{\star} P<0.05,{ }^{* \star} P<0.01,{ }^{* * *} P<0.001$; one-way ANOVA followed by Bonferroni's multiple comparison test.

\section{METHODS}

Mice and reagents. Five-week-old specific pathogen-free female $\mathrm{C} 3 \mathrm{H} / \mathrm{HeOuJ}$ mice were purchased from Charles River (Saint-Germain-sur-l'Abresle, France) and maintained under barrier conditions in filter-topped microloan cages with wood-chip bedding, at a mean temperature of $23 \pm 2{ }^{\circ} \mathrm{C}, 50-55 \%$ relative humidity, and $12 \mathrm{~h}$ light/dark cycle. Acidified drinking water and laboratory food pellets were provided ad libitum. Experiments were approved by the Animal Experiments Committee of the Utrecht University (AEC 2013.III.01.007; 2013.III.10.069; 2014.III.10.087).

Sweet whey protein concentrate 60 (indicated as whey) was obtained from Milei (Milei GmbH, Leutkirch, Germany). CT was purchased from List Biological Laboratories (Campbell, CA), and DON was obtained from Sigma-Aldrich (Zwijndrecht, The Netherlands), catalog number D0156; Lot 044M4042V, purity $\geq 98 \%$.

Treatment protocols. Mice $(n=8)$ were sensitized by intragastric gavage with $20 \mathrm{mg}$ whey mixed with $15 \mu \mathrm{g}$ CT or $100 \mu \mathrm{g}$ DON in $0.5 \mathrm{ml}$ phosphate-buffered saline (PBS) once a week for 5 consecutive weeks. A dosage of $100 \mu \mathrm{g}$ per mouse $\left(\sim 5 \mathrm{mg} \mathrm{kg}^{-1} \mathrm{BW}\right)$ is chosen, to prevent severe side effects because of repeated exposure. On day 35 , mice were challenged intradermally in the ear pinnae of both ears with $10 \mu \mathrm{g}$ of whey in $20 \mu \mathrm{l}$ of PBS to assess the acute allergic skin response as previously described by Schouten et al. ${ }^{28}$ The body temperature was monitored 30,60, and $90 \mathrm{~min}$ after intradermal challenge using a rectal probe; and the anaphylactic shock severity was scored with the scoring table from Li et al ${ }^{29}$ after $45 \mathrm{~min}$ (Supplementary Table S1). Mice received an intragastric challenge with $50 \mathrm{mg}$ whey in $0.5 \mathrm{ml} \mathrm{PBS}$ at day 37 and blood was collected after $30 \mathrm{~min}$ for measurement of MCPT-1. Mice were killed one day later.

In another set of experiments, mice $(n=4)$ were exposed to $20 \mathrm{mg}$ whey alone or to $20 \mathrm{mg}$ whey mixed with $15 \mu \mathrm{g}$ CT or $100 \mu \mathrm{g}$ DON by intragastric gavage and killed after $24 \mathrm{~h}$. For intestinal gene expression analysis, mice $(n=6)$ were exposed to either $15 \mu \mathrm{g}$ CT or $100 \mu \mathrm{g}$ DON dissolved in PBS (vehicle) by intragastric gavage and killed after $6 \mathrm{~h}$, comparable to Akbari et al. ${ }^{11}$

Isolation of intestinal lymphocytes. IEL and LPL were isolated as described previously. ${ }^{7}$ In brief, the small intestine was removed from mice, and fecal contents were flushed with HBBS (Invitrogen, Carlsbad, CA). After carefully removing the PPs, the small intestine was cut into $1-\mathrm{cm}$ pieces, and shaken vigorously in HBBS/EDTA. The mixture was passed through a sieve, supernatant was collected, and the remaining tissue was shaken once more. The remaining tissue was used to isolate LPL, whereas both supernatant fractions were pooled, washed once, followed by isolation of IEL using a Percoll gradient (GE Healthcare, Fisher Scientific, Landsmeer, The Netherlands). For LPL isolation, the remaining tissue was washed and digested with collagenase/DNAse (Roche, Woerden, The Netherlands/ Sigma, Zwijndrecht, The Netherlands). Cells were collected via a 
$100-\mu \mathrm{m}$ strainer and tissue digestion was repeated. Both cell fractions were pooled and washed once. Thereafter, LPL cells were further enriched by a Percoll gradient (GE Healthcare). The collected PP were shaken in HBBS/EDTA, washed once, cut into small fragments, and digested at $37^{\circ} \mathrm{C}$ with collagenase/DNAse (Roche/Sigma). Cells were passed through a $70-\mu \mathrm{m}$ strainer to obtain single cells. Lymphocytes from the MLN and spleen were obtained by squeezing the organs through a $70-\mu \mathrm{m}$ strainer, after which single cells were washed once. To remove the erythrocytes from the spleen, cells were incubated with erylysis buffer (containing $\mathrm{NH}_{4} \mathrm{Cl}, \mathrm{KHCO}_{3}$, and $\mathrm{Na}_{2} \mathrm{EDTA}$ ) for $1 \mathrm{~min}$, and washed once in PBS.

Flow cytometry. For flow-cytometric analysis, cells were first stained with LIVE/DEAD fixable near-IR stain (Molecular Probes, Invitrogen, Carlsbad, CA), followed by incubation with anti-CD16/CD32 (clone 2.4G2) to block the FcR, stained with fluorescent-labeled antibodies and stored in $0.4 \%$ formaldehyde until acquisition. The following antibodies were used (obtained from e-Bioscience (San Diego, CA), Biolegend (San Diego, CA), BD Pharmingen, San Diego, CA, or MD Bioproducts, Zurich, Switzerland): anti-CD3 FITC (145-2C11); antiCD8 $\alpha$ PerCP (53-6.7); anti-CD8 $\beta$ PE (H35-17.2); anti-CD11b FITC (M1/70); anti-CD11c APC (N418); anti-CD45.2 Pacific Blue (104); antiCD86 PerCP (GL-1); anti-CD103 PE (2E7); anti-CD127 APC (A7R34); anti-CD252 (OX40L) PE (RM134L); anti-MHCII PerCP (M5/114.15.2); anti-ST2 PE (DJ8); anti-TCR $\beta$ FITC (H57-597); and anti-TCR $\delta$ APC (GL3). FITC-labeled lineage mixture (Biolegend) contained anti-CD3 (145-2C11), anti-Ly-6G/Ly-6C (RB6-8C5), anti-CD11b (M1/70), antiCD45R/B220 (RA3-6B2), and anti-erythroid cells (TER-119). Data were acquired by means of FACS Canto-II or Accuri C6 (Becton Dickinson, Erembodegem, Belgium), and analyzed using Weasel (The Walter and Eliza Hall Institute of Medical Research, Melbourne, Australia).

Serum immunoglobulins and MCPT-1. Levels of whey-specific immunoglobulins in serum were measured by ELISA as previously described. $^{30}$ MCPT-1 concentrations in serum samples collected $30 \mathrm{~min}$ after oral challenge were measured using an ELISA kit (e-Bioscience), according to the protocol of the manufacturer.

Cell culture and cytokine measurement. Spleen single-cell suspensions $\left(4 \times 10^{6}\right.$ per $\left.\mathrm{ml}\right)$ were incubated in the presence or absence of $50 \mu \mathrm{g} \mathrm{ml}^{-1}$ whey for $96 \mathrm{~h}$ at $37^{\circ} \mathrm{C}$ in a volume of $200 \mu \mathrm{l}$, after which culture supernatants were harvested and stored at $-20{ }^{\circ} \mathrm{C}$ until analysis. Levels of IFN- $\gamma$, IL-5, and IL-13 in culture supernatants were determined by sandwich ELISA according to the instructions of the manufacturer (e-Bioscience).

ST2. Serum samples were collected 6 and $24 \mathrm{~h}$ after each intragastric treatment during sensitization, and soluble ST2 was determined using an ELISA kit (R\&D Systems, Minneapolis, MN) according to the instructions of the manufacturer.

Quantitative reverse transcriptase-PCR for RNA expression. Approximately $50 \mathrm{mg}$ of duodenum and ileum of small intestine (without PPs) was dissected and stored at $-80^{\circ} \mathrm{C}$ until further use. The tissue was suspended into $350 \mu \mathrm{l}$ RNA lysis buffer with $\beta$-mercaptoethanol and homogenized using a TissueLyser (Qiagen, Hilden, Germany) for $1 \mathrm{~min}$ at $25 \mathrm{~Hz}$. Total RNA was isolated using spin columns (Promega, Leiden, The Netherlands) according to the manufacturer's instructions. RNA amounts were determined spectrophotometrically, and RNA purity was measured by NanoDrop 2000 (Thermo Scientific, Waltham, MA) as A260/A280 ratio with expected values between 1.8 and 2. Subsequently, RNA was transcribed into complementary DNA using the iScript cDNA Synthesis Kit (Bio-Rad, Veenendaal, The Netherlands) according to the manufacturer's protocol. Quantitative reverse transcriptase-PCR was performed using a iCycler iQ system (Bio-Rad), and amplification was done using IQ SYBR Green Supermix (Bio-Rad) with $0.3 \mu \mathrm{M}$ final primer concentration.

Forward and reverse primers for claudin1 (CLDN1), claudin2 (CLDN2), claudin3 (CLDN3), claudin4 (CLDN4), occludin (OCLN), zona occludens protein-1 (ZO1), E-cadherin, IL-33, high-mobility group box 1 (HMGB-1), RAE-1, and $\beta$-actin (ACTB) (Table 1) were derived from the US National Center for Biotechnology Information (NCBI; Bethesda, MD) GenBank and were manufactured commercially (Eurogentec, Seraing, Belgium). For each sample, mRNA expression was normalized for the detected Ct value of $\beta$-actin.

Western blot analysis. Approximately $50 \mathrm{mg}$ of duodenal samples (adjacent to samples for Quantitative reverse transcriptase-PCR and without PPs) was dissected and stored at $-80^{\circ} \mathrm{C}$ until further use. The tissue was lysed using $500 \mu \mathrm{l}$ RIPA lysis buffer (Thermo Scientific, Rockford, IL) with protease inhibitors (Roche Applied Science, Penzberg, Germany), and the total protein concentration was measured by the BCA protein assay kit (Thermo Scientific). Equal protein amounts of heat-denaturated non-reduced samples were separated electrophoretically (CriterionTM Gel, 4-20\% Tris-HCl, Bio-Rad Laboratories, Hercules, CA) and electrotransferred onto polyvinylidene difluoride membranes (Bio-Rad). CLDN3 (1:500, Invitrogen) and E-cadherin (1:1,000, e-Bioscience) served as primary antibodies for overnight incubations at $4{ }^{\circ} \mathrm{C}$, and horseradish peroxidase-conjugated goat anti-rabbit or goat anti-rat (1:2,000, Dako, Glostrup, Denmark) was applied as secondary antibodies for $2 \mathrm{~h}$ at room temperature. Finally, blots were washed in PBST, incubated with ECL Prime Western Blotting Detection Reagent (Amersham Biosciences, Roosendaal, The Netherlands), and digital images were obtained with the ChemiDoc MP imager (Bio-Rad Laboratories, Hercules, CA). Subsequently, the membranes were re-probed with a $\beta$-actin antibody $(1: 4,000$, Cell Signaling, Danvers, MA) to assess the equality of loading. Signal intensities were quantified using the ImageJ 1.47 software (National institutes of Health, Bethesda, MD, 2013), and the protein expression was normalized to $\beta$-actin and expressed as the mean fold change in relation to the control group.

Cell culture and TEER measurement. Human epithelial colorectal adenocarcinoma (Caco-2) cells were obtained from the American Type Tissue Collection (Code HTB-37) (Manassas, VA, passages 89-102) and were cultured in Dulbecco's modified Eagle's minimum essential medium, supplemented with $25 \mathrm{~mm}$ Hepes, $4.5 \mathrm{gl}^{-1}$ glucose (Gibco, Invitrogen, Carlsbad, CA), 10\% (v/v) inactivated fetal calf serum (Gibco), glutamine ( $2 \mathrm{~mm}$, Gibco), 1\% (v/v) non-essential amino acids, penicillin $\left(100 \mathrm{U} \mathrm{ml}^{-1}\right)$ and streptomycin $\left(100 \mu \mathrm{g} \mathrm{ml}^{-1}\right.$, Gibco) at $37^{\circ} \mathrm{C}$ in a humidified atmosphere of $95 \%$ air and $5 \% \mathrm{CO}_{2}$. Cells were seeded at a density of $0.3 \times 10^{5}$ cells into $0.3 \mathrm{~cm}^{2}$ high pore density $(0.4 \mu \mathrm{m})$ inserts with a polyethylene terephthalate membrane (BD Biosciences, San Diego, CA) placed in a 24-well plate. The transwell experiments were started after obtaining a confluent Caco-2 monolayer at days 17-19 of culturing with TEER values in the range of $400 \Omega \mathrm{cm}^{2}$ measured by a Millicell-ERS Voltohmmeter (Millipore, Temecular, CA). The Caco-2 cells were incubated with $1.2 \mu \mathrm{g} \mathrm{ml}^{-1}(4.2 \mu \mathrm{M}) \mathrm{DON}$ or $100 \mu \mathrm{g} \mathrm{ml}^{-1} \mathrm{CT}$ added to both compartments (apical and basolateral) of the transwell insert for $24 \mathrm{~h}$, and TEER measurements were conducted. The results are expressed as a percentage of initial value. A DON concentration of $4.2 \mu \mathrm{M}$ was chosen based on the data by Sergent et al, ${ }^{31}$ who described that concentrations of $0.5-7 \mu \mathrm{M}$ DON represent plausible DON concentrations in gastrointestinal tract after ingestion of moderately to highly DON-contaminated food.

$\beta$-Lactoglobulin-FITC flux in Caco-2 cell culture. $\beta$-Lactoglobulin was conjugated with amine-reactive FITC according to the manufacturer's instructions (Sigma). Unbound dyes were separated by column chromatography. The paracellular flux of FITC-labeled $\beta$-lactoglobulin was measured by adding this labeled protein at a concentration of $100 \mu \mathrm{g} \mathrm{ml}^{-1}$ to the apical compartment $(350 \mu \mathrm{l})$ of the transwell plate. Medium at the basolateral compartment was collected after $4 \mathrm{~h}$ and the amount of FITC-labeled $\beta$-lactoglobulin was determined by measuring the fluorescence intensity using a fluorometer (FLUOstar OPTIMA, BMG LABTECH, Offenburg, 
Table 1 Primer sequences of mouse genes used for qRT-PCR analysis

\begin{tabular}{|c|c|c|c|c|}
\hline Target gene & \multicolumn{2}{|c|}{ Primer sequence $\left(5^{\prime}-3^{\prime}\right)$} & AT & Reference \\
\hline CLDN1 & TCTACGAGGGACTGTGGATG & TCAGATTCAGCTAGGAGTCG & 57 & NM_016674.4 \\
\hline CLDN3 & AAGCCGAATGGACAAAGAA & CTGGCAAGTAGCTGCAGTG & 58.7 & NM_009902.4 \\
\hline CLDN4 & CGCTACTCTTGCCATTACG & ACTCAGCACACCATGACTTG & 55 & NM_009903.2 \\
\hline ZO1 & CGAGGCATCATCCCAAATAAGAAC & TCCAGAAGTCTGCCCGATCAC & 58.7 & NM_009386.2 \\
\hline E-cadherin & ACTGTGAAGGGACGGTCAAC & GGAGCAGCAGGATCAGAATC & 64.3 & NM_009864.2 \\
\hline IL-33 & GGTGTGGATGGGAAGAAGCTG & GAGGACTIITGTGAAGGACG & 61 & NM_001164724.1 \\
\hline RAE-1 & CTAGTGCCACCTGGGAATTCA & CATCATTAGCTGATCTCCAGCTCA & 61 & NM_020030.2 \\
\hline HMGB-1 & CTTCGGCCTTCTTCTTGTTCT & GGCAGCTITCTTCTCATAGGG & 61 & NM_010439.3 \\
\hline
\end{tabular}

Abbreviations: ACTB, $\beta$-actin; AT, annealing temperature $\left({ }^{\circ} \mathrm{C}\right)$; CLDN, claudin; HMGB-1, high-mobility group box-1; OCLN, occludin; qRT-PCR, quantitative reverse transcriptase-PCR; RAE-1, retinoic acid early inducible-1; ZO1, zona occludens protein-1.

Germany) at excitation and emission wavelengths of 485 and $520 \mathrm{~nm}$ and calculated on the basis of a standard curve.

Immunofluorescence staining. Localization of CLDN3, CLDN4, E-cadherin in Caco- 2 cells and MCPT-1 in proximal small intestine was assessed by immunofluorescence microscopy. Inserts with cells were fixed with $10 \%$ formalin for $10 \mathrm{~min}$, washed with PBS, and permeabilized with $0.1 \%$ Triton-X-100 for $5 \mathrm{~min}$. The proximal small intestine (4 mice per group) was fixed in 10\% formalin and embedded in paraffin as a "Swiss roll" to permit a complete microscopic examination. After paraffin embedding, $5 \mu \mathrm{m}$ sections were cut (2-3 sections per mouse). These Swiss-rolled paraffin sections were deparaffinized, endogenous peroxidase activity was blocked with $0.3 \% \mathrm{H}_{2} \mathrm{O}_{2}$ (Merck, Darmstadt, Germany) in methanol for $30 \mathrm{~min}$ and rehydrated in a graded ethanol series to PBS. For antigen retrieval, the slides were boiled in $10 \mathrm{~mm}$ citrate buffer ( $\mathrm{pH}$ 6.0) for $10 \mathrm{~min}$ in a microwave and cooled down to room temperature. After blocking with 5\% serum, the Caco- 2 cells were incubated $(2 \mathrm{~h}$, room temperature) with rabbit anti-CLDN3 (1:50, Invitrogen), rabbit anti-CLDN4 (1:50, Invitrogen), and mouse anti-ECadherin (1:50, e-Biosciences), whereas the proximal small intestine was incubated (overnight) with anti-mouse MCPT-1 (1:50, e-Biosciences), both followed by incubation with Alexa-Fluor conjugated secondary antibodies (Invitrogen). A nuclear counterstaining was performed with Hoechst 33342 (1:2,000, Invitrogen). Samples were washed and mounted with FluorSave Reagent (Calbiochem, Amsterdam, The Netherlands). Immunolocalization of CLDN3, CLDN4, E-cadherin, and MCPT-1 was visualized; and images were taken using the Olympus BX60 fluorescence microscope (Zoeterwoude, The Netherlands) equipped with a Leica DFC450C camera.

Statistics. All data are analyzed using the GraphPad Prism version 6.07 software (San Diego, CA). Data from the mouse studies are presented as means \pm standard error of the mean (s.e.m.). Data from in vitro experiments are presented as means \pm s.e.m. of three independent experiments, each performed in triplicate. One-way ANOVA was performed followed by Bonferroni with selected comparisons as a post hoc test. Before testing, logarithmic transformation was performed on antibody and cytokine data from in vivo studies to achieve normal distribution. Differences were considered as significant when $P$-values were $<0.05$

SUPPLEMENTARY MATERIAL is linked to the online version of the paper at http://www.nature.com/mi

\section{ACKNOWLEDGMENTS}

We thank Rob Bleumink for his technical assistance.

\section{DISCLOSURE}

The authors declared no conflict of interest.

c 2016 Society for Mucosal Immunology

\section{REFERENCES}

1. O'Keefe, A.W. et al. Diagnosis and management of food allergies: new and emerging options: a systematic review. J. Asthma Allergy 7, 141-164 (2014).

2. Berin, M.C. \& Sampson, H.A. Food allergy: an enigmatic epidemic. Trends Immunol. 34, 390-397 (2013).

3. Guichard, A. et al. Cholera toxin disrupts barrier function by inhibiting exocyst-mediated trafficking of host proteins to intestinal cell junctions. Cell Host Microbe 14, 294-305 (2013).

4. Lambrecht, B.N. \& Hammad, H. The airway epithelium in asthma. Nat. Med. 18, 684-692 (2012).

5. Strid, J., Sobolev, O., Zafirova, B., Polic, B. \& Hayday, A. The intraepithelial T cell response to NKG2D-ligands links lymphoid stress surveillance to atopy. Science 334, 1293-1297 (2011).

6. Strid, J. et al. Acute upregulation of an NKG2D ligand promotes rapid reorganization of a local immune compartment with pleiotropic effects on carcinogenesis. Nat Immunol 9, 146-154 (2008).

7. Bol-Schoenmakers, M. et al. Regulation by intestinal gammadelta T cells during establishment of food allergic sensitization in mice. Allergy 66, 331-340 (2011).

8. Frossard, C.P., Asigbetse, K.E., Burger, D. \& Eigenmann, P.A. Gut T cell receptor-gammadelta $(+)$ intraepithelial lymphocytes are activated selectively by cholera toxin to break oral tolerance in mice. Clin. Exp. Immunol. 180, 118-130 (2014).

9. Bol-Schoenmakers, M. et al. Diclofenac enhances allergic responses in a mouse peanut allergy model. Clin. Exp. Allergy 41, 424-433 (2011).

10. Kong, J. etal. Comprehensive metabolomics identifies the alarmin uric acid as a critical signal for the induction of peanut allergy. Allergy 70, 495-505 (2015).

11. Akbari, P. et al. Deoxynivalenol: a trigger for intestinal integrity breakdown. FASEB J. 28, 2414-2429 (2014).

12. Wang, Z., Wu, Q., Kuca, K., Dohnal, V. \& Tian, Z. Deoxynivalenol: signaling pathways and human exposure risk assessment-an update. Arch. Toxicol. 88, 1915-1928 (2014). 
13. Pinton, P. et al. The food contaminant deoxynivalenol, decreases intestinal barrier permeability and reduces claudin expression. Toxicol. Appl. Pharmacol. 237, 41-48 (2009).

14. Mulhern, S.A., Fishman, P.H. \& Spiegel, S. Interaction of the B subunit of cholera toxin with endogenous ganglioside GM1 causes changes in membrane potential of rat thymocytes. J. Membr. Biol. 109, 21-28 (1989).

15. Kouzaki, H., lijima, K., Kobayashi, T., O'Grady, S.M. \& Kita, H. The danger signal, extracellular ATP, is a sensor for an airborne allergen and triggers IL-33 release and innate Th2-type responses. J. Immunol. 186, 4375-4387 (2011).

16. Mirchandani, A.S., Salmond, R.J. \& Liew, F.Y. Interleukin-33 and the function of innate lymphoid cells. Trends Immunol. 33, 389-396 (2012).

17. Halim, T.Y. et al. Group 2 innate lymphoid cells are critical for the initiation of adaptive T helper 2 cell-mediated allergic lung inflammation. Immunity 40 , 425-435 (2014).

18. Licona-Limon, P., Kim, L.K., Palm, N.W. \& Flavell, R.A. TH2, allergy and group 2 innate lymphoid cells. Nat. Immunol. 14, 536-542 (2013).

19. Chu, D.K. et al. IL-33, but not thymic stromal lymphopoietin or IL-25, is central to mite and peanut allergic sensitization. J. Allergy Clin. Immunol. 131, 187-200.e1-8 (2013).

20. Smith, D.E. IL-33: a tissue derived cytokine pathway involved in allergic inflammation and asthma. Clin. Exp. Allergy 40, 200-208 (2009).

21. Chu, D.K. et al. Thelper cell IL-4 drives intestinal Th2 priming to oral peanut antigen, under the control of OX4OL and independent of innate-like lymphocytes. Mucosal Immunol. 7, 1395-1404 (2014).

22. Commission Regulation (EC) No. 1881/2006 setting maximum levels for certain contaminants in foodstuffs. Off. J. Euro. Union L 364, 5-24 (2006).
23. Rodriguez-Carrasco, Y., Molto, J.C., Manes, J. \& Berrada, H. Exposure assessment approach through mycotoxin/creatinine ratio evaluation in urine by GC-MS/MS. Food Chem. Toxicol. 72, 69-75 (2014).

24. Solfrizzo, M., Gambacorta, L. \& Visconti, A. Assessment of multimycotoxin exposure in southern Italy by urinary multi-biomarker determination. Toxins 6, 523-538 (2014).

25. Warth, B et al. Assessment of human deoxynivalenol exposure using an LC-MS/MS based biomarker method. Toxicol. Lett. 211, 85-90 (2012).

26. Wan, L.Y., Turner, P.C. \& El-Nezami, H. Individual and combined cytotoxic effects of Fusarium toxins (deoxynivalenol, nivalenol, zearalenone and fumonisins B1) on swine jejunal epithelial cells. Food Chem. Toxicol. 57, 276-283 (2013).

27. Alassane-Kpembi, I. et al. New insights into mycotoxin mixtures: the toxicity of low doses of Type B trichothecenes on intestinal epithelial cells is synergistic. Toxicol. Appl. Pharmacol. 272, 191-198 (2013).

28. Schouten, B. et al. Oligosaccharide-induced whey-specific CD25(+) regulatory $\mathrm{T}$-cells are involved in the suppression of cow milk allergy in mice. J. Nutr. 140, 835-841 (2010).

29. Li, X.M., Schofield, B.H., Huang, C.K., Kleiner, G.I. \& Sampson, H.A. A murine model of IgE-mediated cow's milk hypersensitivity. J. Allergy Clin. Immuno./ 103, 206-214 (1999).

30. van den Elsen, L.W. et al. Dietary long chain $n-3$ polyunsaturated fatty acids prevent allergic sensitization to cow's milk protein in mice. Clin. Exp. Allergy 43, 798-810 (2013).

31. Sergent, T., Parys, M., Garsou, S., Pussemier, L., Schneider, Y.J. \& Larondelle, Y. Deoxynivalenol transport across human intestinal Caco-2 cells and its effects on cellular metabolism at realistic intestinal concentrations. Toxicol. Lett. 164, 167-176 (2006). 